\title{
Strongly decelerated expansion of SN 1979C
}

\author{
J. M. Marcaide ${ }^{1}$, M. A. Pérez-Torres ${ }^{1,2}$, E. Ros ${ }^{3}$, A. Alberdi ${ }^{4}$, P. J. Diamond ${ }^{5}$, J. C. Guirado ${ }^{1}$, L. Lara ${ }^{4}$, \\ S. D. Van Dyk ${ }^{6}$, and K. W. Weiler ${ }^{7}$ \\ 1 Departamento de Astronomía, Universitat de València, 46100 Burjassot, Spain \\ 2 Istituto di Radioastronomia/CNR, via P. Gobetti 101, 40129 Bologna, Italy \\ 3 Max-Planck-Institut für Radioastronomie, Auf dem Hügel 69, 53121 Bonn, Germany \\ 4 Instituto de Astrofísica de Andalucía, CSIC, Apdo. Correos 3004, 18080 Granada, Spain \\ 5 MERLIN/VLBI National Facility, Jodrell Bank Observatory, Macclesfield, Cheshire SK11 9DL, UK \\ 6 Infrared Processing and Analysis Center, California Institute of Technology, Mail Code 100-22, Pasadena, \\ CA 91125, USA \\ 7 Remote Sensing Division, Naval Research Laboratory, Code 7213, Washington, DC 20375-5320, USA
}

Received 20 July 2001 / Accepted 11 December 2001

\begin{abstract}
We observed SN 1979C in M100 on 4 June 1999, about twenty years after explosion, with a very sensitive four-antenna VLBI array at the wavelength of $\lambda 18 \mathrm{~cm}$. The distance to M100 and the expansion velocities are such that the supernova cannot be fully resolved by our Earth-wide array. Model-dependent sizes for the source have been determined and compared with previous results. We conclude that the supernova shock was initially in free expansion for $6 \pm 2 \mathrm{yrs}$ and then experienced a very strong deceleration. The onset of deceleration took place a few years before the abrupt trend change in the integrated radio flux density curves. We estimate the shocked swept-up mass to be $M_{\mathrm{sw}} \sim 1.6 M_{\odot}$, assuming a standard density profile for the CSM. Such a swept-up mass for SN 1979C suggests a mass of the hydrogen-rich envelope ejected at explosion no larger than $M_{\text {env }} \sim 0.9 M_{\odot}$. If SN 1979C originated in a binary star, the low value of $M_{\text {env }}$ suggests that the companion of the progenitor star stripped off most of the hydrogen-rich envelope mass of the presupernova star prior to the explosion.
\end{abstract}

Key words. techniques: interferometric - supernovae: individual: SN 1979C - ISM: supernova remnants radio continuum: stars - galaxies: individual: M100

\section{Introduction}

Supernova SN 1979C in M100 was discovered on 1979 April 19 by Gus E. Johnson (Mattei et al. 1979) at around magnitude 12 in the visible, and it is thought to have exploded on April 4, 1979 (Weiler et al. 1986). Optical spectra first showed a featureless continuum, which later evolved to exhibit strong $\mathrm{H} \alpha$ emission (e.g., Schlegel 1996). SN 1979C was extraordinarily luminous, $M_{B}^{\max } \approx-20$ (e.g., Young \& Branch 1989), making it among the most luminous type II supernova ever observed. SN 1979C was also found to be a radio supernova (RSN) (Weiler \& Sramek 1980). Indeed, at a distance of $16.1 \pm 1.3 \mathrm{Mpc}$ estimated for M100 (Ferrarese et al. 1996) it is one of the intrinsically strongest RSN (Weiler et al. 1996). From $\mathrm{H} \alpha$ widths an expansion speed of $9200 \pm 500 \mathrm{~km} \mathrm{~s}^{-1}$ was estimated (Panagia et al. 1980 and G. Vettolani, priv. comm.) for epoch around $45 \mathrm{~d}$.

Send offprint requests to: J. M. Marcaide, e-mail: J.M.Marcaide@uv.es
SN 1979C has been classified as a SN of type II-L. Fesen et al. (1999), hereafter F99, have studied the latetime optical properties of SN $1979 \mathrm{C}$ and other three type II-L supernovae. Late-time optical emission lines from type SNe II-L appear surprisingly steady over long time intervals. These authors find similar expansion velocities in the range $5100-6000 \mathrm{~km} \mathrm{~s}^{-1}$ in the strongest lines present (H $\alpha$, [OI] 6300, 6364 $\AA$, [OII] 7319, $7330 \AA$, and [OIII] 4959, $5007 \AA$ ) for all type II-L supernovae. From the asymmetries of the lines these authors find evidence of dust formation. Strong radio emission from a SN appears to be strongly correlated with late time bright optical emission, with SN 1979C as a prime example. In SN 1979C, all detected lines but $\mathrm{H} \alpha$ show emission peaks at -5000 and $-1000 \mathrm{~km} \mathrm{~s}^{-1}$ suggestive of clumpy emission from material coming mostly from ejecta, and physically separated from the material emitting $\mathrm{H} \alpha$. The $\mathrm{H} \alpha$ emission tracks well the peak $6 \mathrm{~cm}$ emission (Weiler et al. 1991) indicating that both emissions are related to the material from the swept-up shell. 


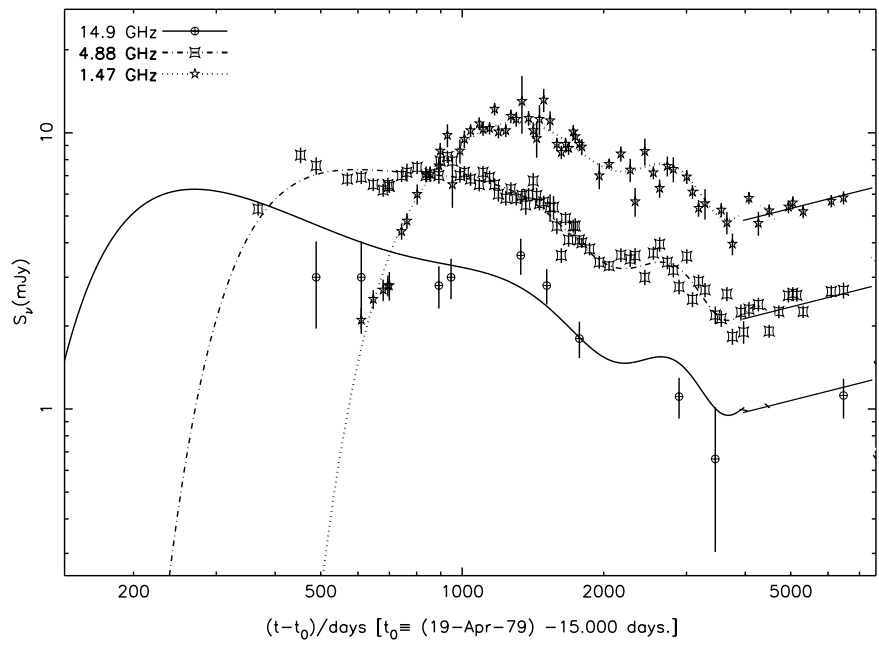

Fig. 1. Radio light curves for SN 1979 C in M100 at wavelengths of 20,6 , and $2 \mathrm{~cm}$. The curves represent the best-fit model light curves. The best-fit parameters were determined using only data through 1990 December (day 4300). Notice the flux density increase from day 4000 onwards (Montes et al. 2000).

The evolution of the radio emission from SN 1979C has been interpreted within the mini-shell model (Chevalier 1982) as due to synchrotron emission from the outer part of the shock swept-up shell of circumstellar material attenuated by thermal absorption from the even more distant ionized CSM (Weiler et al. 1986; Montes et al. 2000). Synchrotron self absorption which has been shown to be relevant in SN 1993J (Fransson \& Björnsson 1998; Pérez-Torres et al. 2001) might also play a role in SN 1979C, although Chevalier (1998) is not of this opinion. The modulation present in the radio light curves of SN 1979C has led Weiler et al. (1992) and Montes et al. (2000) to model the progenitor as possibly being in a detached eccentric binary system with a less massive companion. From a study of the emission environment of SN 1979C, Van Dyk et al. (1999) have estimated the mass of the progenitor to be $17-18( \pm 3) M_{\odot}$. Hydro-dynamical simulations by Schwarz \& Pringle (1996) confirm that a binary system similar to that proposed by Weiler et al. (1992) with a spiral shaped stellar wind around the progenitor is feasible for SN 1979C. Such modulation of the density of the gas surrounding the RSN and now being shocked, might have dramatic effects on the shape of the radio structure. A determination of such radio structure would be a powerful way to further understand the presupernova phase of the progenitor of SN 1979C.

The radio emission of this RSN, monitored by Montes et al. (2000), has started to show an interesting trend which can be seen in Fig. 1: after years of steady decline the radio emission has been constant, or perhaps increasing, since about $1990(\sim 10 \mathrm{yr})$ in a way somewhat reminiscent of the radio emission increase for the case of SN 1987A (Ball et al. 1995). It appears that after a standard RSN radio emission decline phase (Weiler et al. 1996) the expanding shock may have encountered denser ionized gas which has boosted the radio emission. These results may be in accord with those of F99 which show that high electron densities $\left(10^{5-7} \mathrm{~cm}^{-3}\right)$ are necessary to explain the absence of several lines, such as [OII] 3726, $3729 \AA$ in their optical spectra.

Soft X-rays have been detected from SN 1979C by Immler et al. (1998), Ray et al. (2001), and Kaaret (2001). The soft X-ray luminosity appears constant or slightly declining from epoch $16 \mathrm{yr}$ through $20 \mathrm{yr}$. This result appears compatible with the model predictions of circumstellar interactions (Chevalier \& Fransson 1994).

VLBI observations at 3.6, 6, 13 and $18 \mathrm{~cm}$ by Bartel et al. (1985), hereafter B85, determined a size of SN 1979C and its growth for epochs ranging from Dec. 1982 to May 1984 assuming a synchrotron emitting optically thin spherical model. At those early epochs the source could not be resolved by any VLBI array. At an average expansion speed of, for example, $\sim 8000 \mathrm{~km} \mathrm{~s}^{-1}$, one should expect a shell radius growth rate of $\sim 0.3 \mu$ as day $^{-1}$ which over 20 years should yield a shell radius of $\sim 2.2$ mas which could be resolved by $\lambda 6 \mathrm{~cm}$ VLBI if detected on the long baselines. However, due to sensitivity limitations of the available arrays, the chances of detecting it at $\lambda 6 \mathrm{~cm}$ at the present time are null. On the other hand, at $\lambda 18 \mathrm{~cm}$ the emission strength of SN 1979C and the sensitivity of the arrays make the detection possible. We have carried out state-of-the-art $\lambda 18 \mathrm{~cm}$ observations in an attempt to learn as much as possible about the source structure.

\section{Observations}

SN 1979C was observed on 4 June 1999 from 19:30UT to $01: 30 \mathrm{UT}$ at $1.6 \mathrm{GHz}$ with a sensitive transatlantic VLBI array. The antennas used were (diameter, location): Effelsberg (100 m, Germany), Robledo (70 m, Spain), phased-VLA (130 m-equivalent, NM, US), and Goldstone (70 m, CA, US). Data were recorded using a standard VLBA mode with a data rate of $256 \mathrm{Mb} / \mathrm{s}$, covering a bandwidth of $64 \mathrm{MHz}$. The data were correlated at the VLBA Correlator Center of the National Radio Astronomy Observatory (NRAO) ${ }^{1}$ in Socorro, NM, US.

The observations were made with a duty cycle of about 26 min consisting of 3 min observations of the calibrator source PKS B1157+156, followed by 5 min observations of the calibrator source TXS $1214+161$, in turn followed by $14 \mathrm{~min}$ observations of the source SN 1979C. The source 3C 274 was observed twice as a calibrator, once at the beginning and once at the end of the observations.

The correlated data were analyzed using the Astronomical Image Processing System (AIPS). A basic integration time of $2 \mathrm{~s}$ was used. The phase slopes across each of the $8 \mathrm{MHz}$ bands and over the $64 \mathrm{MHz}$ synthesized band were aligned using a phase calibration determined from observations of the calibrator source 3C 274. The data of the calibrators PKSB1157+156

1 NRAO is operated under cooperative agreement with the National Science Foundation. 
and TXS 1214+161 were then fringe-fitted in a standard manner. In the next step, phases, delays, and delayrates corresponding to the sources PKS B1157+156 and TXS 1214+161 were interpolated to the source SN 1979C, to provide an initial model for the fringing of the data of this radio source. A solution was obtained by fringing the data using narrow search windows set around the initial model. Fringe detections of SN 1979C were obtained for all baselines at almost all times. However, some SN 1979C data had to be removed from the analysis after visual inspection of the data of the two main calibrators. Indeed, for some time ranges in the observations there were calibration problems or data with lower than acceptable quality. For instance, high winds at the phased-VLA produced an auto-stowing of some antennas and problems in the phasing during the second half of the observations. Therefore, data from the VLA from 23:30 UT to 01:15 UT were edited.

Adjacent to the VLBI observations reported in this paper, some cross-scans on PKS B1157+156 were carried out at $1.6 \mathrm{GHz}$ to fix the pointing parameters of the antenna in Effelsberg. As a byproduct, those observations allowed the total flux density of PKSB1157+156 to be measured as $0.79 \pm 0.02 \mathrm{Jy}$ (A. Kraus, priv. comm.). This value was adopted to set our flux density scale. Then, by readjusting the gains of the antennas, the VLBI map of PKS B1157+156 was forced to have the same flux density as the total flux density (that is, PKS B1157+156 was assumed unresolved by our VLBI array). With this calibration procedure for the antennas of the array a total flux density of $5.4 \pm 0.2 \mathrm{mJy}$ was obtained for SN $1979 \mathrm{C}$.

Further model fitting analysis was carried out with MODELFIT in DIFMAP (Shepherd et al. 1994), and with QFIT in the Caltech VLBI Analysis Package (Pearson \& Readhead 1984).

\section{The size of SN 1979 C}

We assumed spherical symmetry in the modeling. The data remaining after the editing were of the highest quality, but from a look at the data it was not fully clear that all points in the visibility vs. uv-radius plot are on the main lobe of the interferometric response. We had to prove to ourselves that such was indeed the case. Good measurements of the closure phases (all being around zero and none around $180^{\circ}$ ) were a great help. We conducted several tests and model fits to convince ourselves that indeed the array at $\lambda 18 \mathrm{~cm}$ was not large enough to be able to resolve the structure of SN 1979C. Unfortunately, there are then many models which can reproduce the data equally well (see Marscher 1985 and B85).

Thus, we decided to determine the size of SN 1979C for the following putative source shapes: an optically thick source, an optically thin shell of width $30 \%$ of the external radius, and a ring that is an infinitely bright shell of zero width. The last one is obviously unphysical and its determination is only valuable as a limit. Even the first of the three putative source shapes is not expected

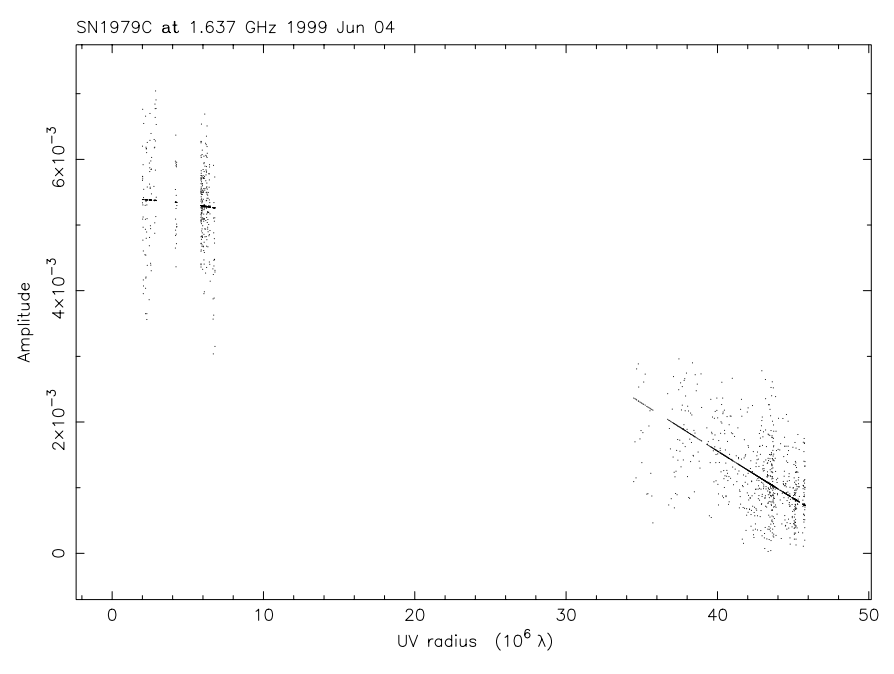

Fig. 2. Visibility amplitude versus interferometric distance. The model (solid line) of 3.60 mas outer diameter shell with a $30 \%$ thickness is plotted superimposed on the data.

given that the spectrum of the source is optically thin with $S \propto \nu^{-0.63 \pm 0.03}$ (Montes et al. 2000). The diameters determined by model fitting from our data for such models are $4.57 \pm 0.25,3.60 \pm 0.17$, and $3.10 \pm 0.14$ mas, respectively. The uncertainties were approximately estimated from the statistical errors given by the programs QFIT and MODELFIT, taking into account discrepant results yielded by them.

A fit for the preferred model of an optically thin shell of diameter $3.60 \pm 0.17$ mas with width $30 \%$ of the external radius is shown, along with the measured visibilities, as the solid line in Fig. 2.

\section{Discussion}

Marcaide et al. (1995a, 1995b, 1997) determined for SN 1993J a shell structure with a shell width $30 \%$ of the outer radius, somewhat larger than that predicted by Chevalier \& Fransson (1994). Therefore, we have adopted a shell of width $30 \%$ of the external radius as a model for SN 1979C in this paper and all our discussion will be based on such model. For the present discussion all previous results by $\mathrm{B} 85$ will be transformed (normalized) to this model.

At a distance of $16.1 \mathrm{Mpc}$ to M100 an angular size of 1 mas corresponds to a linear size of $2.41 \times 10^{17} \mathrm{~cm}$. Hence, the source outer radius (1.80 mas) corresponds to $4.33 \times 10^{17} \mathrm{~cm}$. Since $20.12 \mathrm{yr}$ have elapsed since the explosion, the corresponding average supernova expansion velocity is $\sim 6800 \mathrm{~km} \mathrm{~s}^{-1}$. Since early expansion speeds were estimated at epoch $45 \mathrm{~d}$ from the $\mathrm{H} \alpha$ lines as $~ 9200 \mathrm{~km} \mathrm{~s}^{-1}$ it follows that the expansion has been strongly decelerated by the action of the circumstellar matter. How strongly? It is difficult to make a detailed analysis to answer such a question, since intermediate size estimates are not available. Nonetheless, we now discuss our VLBI results, in conjunction with previously published VLBI and optical 
spectroscopy data, to show that SN 1979C has indeed suffered a heavy deceleration in its expansion, starting $6 \pm 2 \mathrm{yr}$ after its explosion.

B85 determined for SN1979C a diameter of $2.16_{-0.50}^{+0.44}$ mas $5.17 \mathrm{yr}$ after explosion, from $1.67 \mathrm{GHz}$ VLBI observations using an optically thin uniform sphere model. This value translates into a diameter of $1.56_{-0.36}^{+0.31}$ mas for our discussion shell model. This size corresponds to an average speed for the first $5.17 \mathrm{yr}$ of $\sim 11500 \mathrm{~km} \mathrm{~s}^{-1}$. On the other hand, the average speed of the supernova over the first six weeks after explosion as determined from the widths of $\mathrm{H} \alpha$ lines was $\sim 9200 \pm 500 \mathrm{~km} \mathrm{~s}^{-1}$ (Panagia et al. 1980; G. Vettolani, priv. comm.), which is about $20 \%$ lower than the average velocity estimated for the first 5 years from the B85 estimates normalized to our discussion model. These two results are nicely consistent. Indeed, in the mini-shell model of Chevalier (1982), the radio emission comes from the forward shock, while the optical, UV, and X-ray emissions come mainly from the reverse shock, which moves slower than the forward shock. Hence the velocity ratio determined from the VLBI and optical observations is roughly what is expected for the radii ratio of the forward and reverse shocks, in a model of $20-30 \%$ shell width (Chevalier \& Fransson 1994; Marcaide et al. 1997) if SN 1979C followed a free, self-similar expansion for the first 5 years after its explosion. A free expansion for the first 4 years, followed by a decelerated expansion would also be compatible with the mentioned results. These results should be understood as evidence of free expansion of SN 1979 C for the first $4-5$ years and of the presence of a shell-like structure, and not as evidence of a given shell width, since different model normalizations of the results of B85 would yield equally consistent results and a similar conclusion.

An estimate of $m\left(R \propto t^{m}\right)$ using the size estimate of B85 and our size estimate results in $m=0.62_{-0.17}^{+0.22}$. This result shows that SN $1979 \mathrm{C}$ has indeed dramatically decreased its growth rate after a likely initial phase of free expansion ( $m \approx 1.0$; see Fig. 3 ). The optical data, although as scarce as the VLBI data, can be used as a check of the reliability of our estimate of $m$. In a self-similar expansion scenario $R \propto t^{m}$ and, consequently, $v \propto t^{m-1}$. If we now assume that the supernova did not decelerate for the first 5.17 years, we can take the value of $9200 \mathrm{~km} \mathrm{~s}^{-1}$ as the expansion velocity just prior to the phase of strongly decelerated expansion. Combining this value with that of $6200 \pm 300 \mathrm{~km} \mathrm{~s}^{-1}$ at epoch 14.09 yr (F99 and R. Fesen, priv. comm.), gives $m=0.61_{-0.11}^{+0.10}$, in agreement with our estimate of $m=0.62_{-0.17}^{+0.22}$.

Hence, $m=0.62$ appears to be an adequate value to characterize a phase of strong deceleration in the expansion of SN 1979C, assuming it started about 5.17 years after its explosion. If the supernova started to decelerate later than $5.17 \mathrm{yr}$, then $m$ should be smaller, and correspondingly stronger the deceleration of SN 1979C. It seems somewhat arbitrary to assume that the deceleration set in

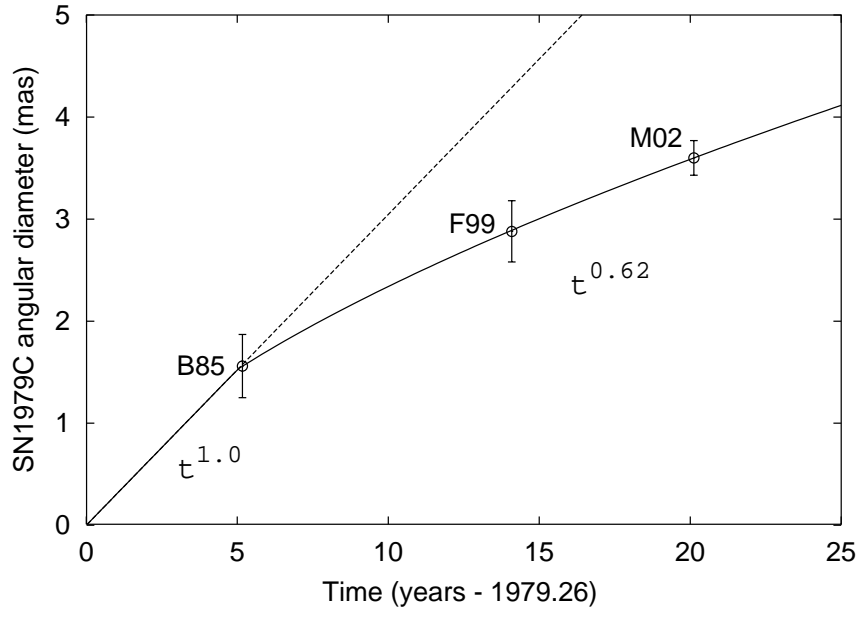

Fig. 3. Angular diameter vs. age of SN 1979C. B85 indicates the size estimate from Bartel et al. (1985) normalized to our model (see text), F99, the size estimated by us based on the optical results by Fesen et al. (1999), and M02 indicates our results. The solid line indicates a possible expansion, which is free for the first 5 yrs and decelerated from then on. The size estimate F99 shown is compatible with this expansion scenario and it would be slightly different for scenarios where the free expansion would last much longer than $5 \mathrm{yr}$, which is not the case. See text.

at the same time as the observations of B85. Indeed, there is nothing apparent in the integrated radio light curves which could point to a change at that particular epoch. It is intriguing, though, that the integrated radio light curves of SN 1979C (Fig. 1) seem to show an increase in flux at around $t=2200$ days, or $6 \mathrm{yr}$ after its explosion. One could speculate that the flux rise at this epoch is somehow related to a change in the deceleration parameter, likely due to the shock front of the supernova entering a high-density region of the circumstellar medium (CSM).

Our estimate of the angular diameter for SN 1979C can also be used to obtain an upper limit for the epoch at which the phase of strong deceleration started. We find that the latest epoch of free expansion which is still compatible with both the optical observations of F99 (epoch $14.09 \mathrm{yr}$ ) and our VLBI determination at $20.12 \mathrm{yr}$, and with the fact that a lower limit is set by SN 1979C entering a Sedov phase $(m=0.4)$, corresponds to $\sim 8 \mathrm{yr}$.

The integrated radio emission curves have been well modeled for the first 10 years by Weiler et al. (1992) and Montes et al. (2000) who assumed self-similar expansion and a modulation in the density of the CSM due to the binary interaction, to explain the modulation in the flux density curve. Montes et al. (2000) found that starting around epoch $10 \mathrm{yr}$ the radio emission started to grow after a long decline predicted by the radio emission model. Can our VLBI observations shed any light on what happened at epoch $10 \mathrm{yr}$ ? The VLBI results by B85 and us require a strong deceleration of the expansion. Such deceleration is compatible with the optical results by F99 for a self-similar expansion. Our results rule out any initial 
free expansion longer than 8 years. Thus, the onset of decelerated expansion took place well before the epoch $10 \mathrm{yr}$ where the radio emission decline turned onto a slight growth. The sooner the onset of the decelerated expansion the milder the deceleration needed to be compatible with the observations. The epoch of that onset is restricted to the range 4-8 years and the corresponding deceleration parameter $m$ to the range $0.67-0.44$. (Strictly speaking an onset at 3 years with a corresponding $m=0.72$ is not excluded, but it appears unlikely since it yields a radio to optical velocity ratio of 0.68 at epoch $5.17 \mathrm{yr}$. Such a ratio is small, but not yet inconsistent with the models used. In any case, the deceleration required for an early onset is comparable to the strongest decelerations reported in other cases by Marcaide et al. (1997) for SN 1993J in M 81 and Mc Donald et al. (2001) for 43.31+592 in M82.) An onset of decelerated expansion at epoch $6 \mathrm{yr}$ (corresponding to a deceleration parameter $m=0.57$ ) appears most likely and it may be associated with a small halt in the otherwise decreasing flux density trend, a halt which may be partly related to encountering a denser CSM.

If the expansion of SN 1979C has significantly decelerated, as our VLBI estimate shows, then the mass of the CSM swept up by the shock front, $M_{\mathrm{sw}}$, must be comparable to or larger than the mass of ejected hydrogen-rich envelope, $M_{\text {env }}$. Our estimated angular diameter translates into a forward shock radius of $\sim 4.33 \times 10^{17} \mathrm{~cm}$. For a presupernova wind of velocity $v_{\mathrm{w}}=10 \mathrm{~km} \mathrm{~s}^{-1}$, this radius corresponds to $\sim 13700 \mathrm{yr}$ prior to the explosion of SN 1979C. Lundqvist \& Fransson (1988) and Weiler et al. (1991) estimated a mass-loss rate of $\sim 1.2 \times 10^{-4} M_{\odot} \mathrm{yr}^{-1}$ for the progenitor of SN 1979C. If we now assume that the circumstellar medium up to this distance is a standard one $\left(\rho_{\mathrm{cs}} \propto r^{-2}\right)$, we then obtain $M_{\mathrm{sw}} \sim 1.6 M_{\odot}$. Momentum conservation therefore implies that $M_{\mathrm{env}} \approx 0.59 M_{\mathrm{sw}}$, which results in a mass for the ejected hydrogen-rich envelope of $\sim 0.94 M_{\odot}$.

In the case of the Type II supernova SN1993J, whose progenitor was a $\sim 15 M_{\odot}$ star in a binary system, mass exchange with its companion meant that the mass of the hydrogen-rich envelope ejected at the explosion was 0.2-0.4 $M_{\odot}$ (Woosley et al. 1994; Houck \& Fransson 1996), much less than the $\approx 3.3 M_{\odot}$ one would expect from a single-star model (Höflich et al. 1993). Weiler et al. (1986) have suggested that SN1979C was born in a binary system consisting of a $15-18 M_{\odot}$ red supergiant and a $10 M_{\odot} \mathrm{B} 1$ main-sequence star. If we assume that SN 1979C lost, like SN 1993J, most of its hydrogen-rich envelope prior to explosion, then the $\sim 0.9 M_{\odot}$ decelerated ejecta suggested from our observations represents at least all of the remaining hydrogen-rich envelope.

From VLBI observations of SN 1979C more than 20 years after its explosion, we conclude that an initial free expansion of SN 1979C was followed by a strong deceleration which set on $\sim 6$ yr after the supernova explosion. We estimate the shocked swept-up mass to be $M_{\mathrm{sw}} \sim 1.6 M_{\odot}$, assuming a standard density profile for the CSM. Such a swept-up mass for SN 1979C suggests a mass of the hydrogen-rich envelope ejected at explosion as low as $M_{\text {env }} \sim 0.94 M_{\odot}$ or lower. If, as suggested by Weiler et al. (1986) and Schwarz \& Pringle (1996) SN 1979C originated in a binary star (alike SN 1993J) this value of $M_{\mathrm{env}}$ suggests that the companion of the progenitor star stripped off most of the hydrogen-rich envelope mass of the presupernova star prior to its explosion. New observations at $\lambda 18 \mathrm{~cm}$ around 2005 should be able to test the above conclusion and would also be essential to image the true structure of SN 1979C. On the other hand, a continued rise in the level of flux density at $\lambda 6 \mathrm{~cm}$ and improved VLBI instrumentation and techniques like phase-referencing may allow an earlier determination of the intrinsic structure of SN $1979 \mathrm{C}$ with high resolution and a test of the binary star scenario which the present results tend to favor.

Acknowledgements. We are grateful to A. Kraus for the help provided during the observations and the flux density measurements on PKS B1157+156. This work has been partially supported by the Spanish DGICYT Grants No. PB96-0782 and PB97-1164. NRAO is operated under license by Associated Universities, Inc., under cooperative agreement with NSF. We acknowledge support from the European Commission's TMRLSF programme, Contract No. ERBFMGEST950012. JMM is grateful to the Max-Planck Institut für Radioastronomie for the support received for this research during his visit. KWW wishes to thank the Office of Naval Research for the 6.1 funding supporting this work.

\section{References}

Bartel, N., Rogers, A. E. E., Shapiro, I. I., et al. 1985, Nature, 318, 25 (B85)

Ball, L., Campbell-Wilson, D., Crawford, D. F., \& Turtle, A. J. 1995, ApJ, 453, 864

Chevalier, R. A., \& Fransson, C. J. 1994, ApJ, 420, 268

Chevalier, R. A. 1998, ApJ, 499, 810

Fesen, R. A., Gerardy, C. L., Filippenko, A. V., et al. 1999, AJ, 117, 725 (F99)

Ferrarese, L., Freedman, W. L., Hill, R. J., et al. 1996, ApJ, 464,568

Fransson, C., \& Björnsson, C.-I. 1998, ApJ, 509, 861

Höflich, P., Langer, N., \& Duschinger, M. 1993, A\&A, 275, L29

Houck, J. C., \& Fransson, C. 1996, ApJ, 456, 811

Immler, S., Pietsch, W., \& Aschenbach, B. 1998, A\&A, 331, 601

Kaaret, P. 2001, ApJ, 560, 715

Lundqvist, P., \& Fransson, C. 1988, A\&A, 192, 221

Mc Donald, A. R., Muxlow, T. W. B., Pedlar, A., et al. 2001, MNRAS, 322, 100

Marcaide, J. M., Alberdi, A., Ros, E., et al. 1995a, Nature, 373,44

Marcaide, J. M., Alberdi, A., Ros, E., et al. 1995b, Science, 270,1475

Marcaide, J. M., Alberdi, A., Ros, E., et al. 1997, ApJ, 486, L31

Marscher, A. P. 1985, Nature, 318, 18 
Mattei, J., Johnson, G. E., Rosino, L., Rafanelli, P., \& Weiler, K. W., Sramek, R. A., Panagia, N., van der Hulst, Kirshner, R. 1979, IAU Circ., 3348 J. M., \& Salvati, M. 1986, ApJ, 301, 790

Montes, M. J., Weiler, K. W., Van Dyk, S. D., et al. 2000, ApJ, 532,1124

Weiler, K. W., Van Dyk, S. D., Panagia, N., et al. 1991, ApJ, 380,161

Panagia, N., Vettolani, G., Boksenberg, A., et al. 1980, Weiler, K. W., Van Dyk, S. D., Pringle, J. E., \& Panagia, N. MNRAS, 192, 861

Pearson, T. J., \& Readhead, A. C. S. 1984, ARA\&A, 22, 97

Pérez-Torres, M. A., Alberdi, A., \& Marcaide, J. M. 2001, A\&A, 374, 997

Ray, A., Petre, R., \& Schlegel, E. M. 2001, AJ, 122, 966

Schlegel, E. M. 1996, AJ, 111, 1660

Schwarz, D. H., \& Pringle, J. E. 1996, MNRAS, 282, 1018

Shepherd, M. C., Pearson, T. J., \& Taylor, G. B. 1994, BAAS, 26,287

Weiler, K. W., \& Sramek, R. A. 1980, IAU Circ., 3485 1992, ApJ, 399, 672

Weiler, K. W., Van Dyk, S. D., Sramek, R. A., \& Panagia, N. 1996, in Radio Emission from the Stars and the Sun, ed. A. R. Taylor, \& J. M. Paredes (San Francisco, CA, US), ASP Conf. Ser., 93, 141

Woosley, S. E., Eastman, R. G., Weaver, T. A., et al. 1994, ApJ, 429, 300

Van Dyk, S. D., Peng, C. Y., Barth, A. J., et al. 1999, PASP, 111,313

Young, T. R., \& Branch, D. 1989, ApJ, 342, 79 